\title{
Surgical embryo collection but not nonsurgical embryo transfer compromises postintervention prolificacy in sows
}

\author{
C. A. Martinez, A. Nohalez, I. Parrilla, J. L. Vazquez, J. Roca, C. Cuello, Heriberto \\ Rodriguez-Martinez, E. A. Martinez and M. A. Gil \\ Journal Article
}

Tweet

N.B.: When citing this work, cite the original article.

Original Publication:

C. A. Martinez, A. Nohalez, I. Parrilla, J. L. Vazquez, J. Roca, C. Cuello, Heriberto RodriguezMartinez, E. A. Martinez and M. A. Gil, Surgical embryo collection but not nonsurgical embryo transfer compromises postintervention prolificacy in sows, Theriogenology, 2017. 87, pp.316-320.

http://dx.doi.org/10.1016/j.theriogenology.2016.09.009

Copyright: Elsevier

http://www.elsevier.com/

Postprint available at: Linköping University Electronic Press

http://urn.kb.se/resolve?urn=urn:nbn:se:liu:diva-133249
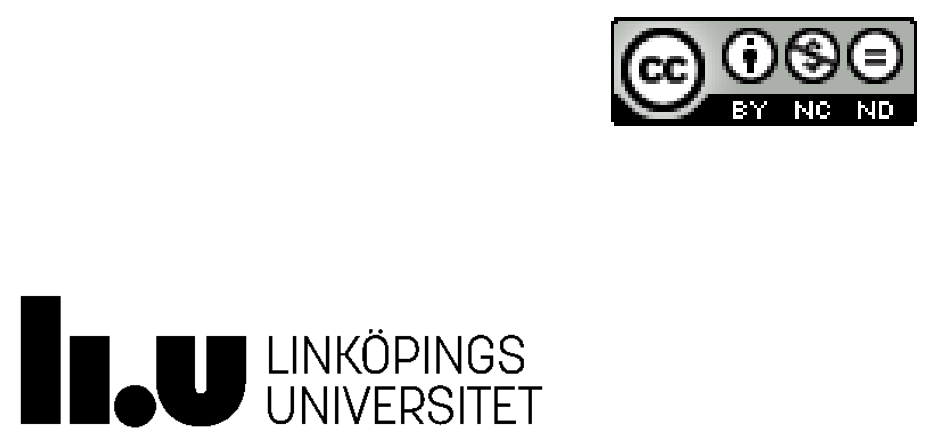
Revised

Surgical embryo collection but not non-surgical embryo transfer compromises post-intervention prolificacy in sows

CA Martinez ${ }^{1}$, A Nohalez ${ }^{1}$, I Parrilla ${ }^{1}$, JL Vazquez ${ }^{1}$, J Roca ${ }^{1}$, C Cuello ${ }^{1}$, H RodriguezMartinez $^{2}$, EA Martinez ${ }^{1}$ and MA Gil ${ }^{1}$

${ }^{1}$ Department of Animal Medicine and Surgery, University of Murcia, Spain; and ${ }^{2}$ Department of Clinical \& Experimental Medicine (IKE), Linköping University, Sweden

Corresponding author. Tel.: +34 868884734; fax: +34 868887069 .

E-mail address: emilio@um.es (E.A. Martinez).

\section{ABSTRACT}

Recent advances in non-surgical deep uterine (NsDU) embryo transfer (ET) technology allow the non-invasive transfer of porcine embryos into recipients, overcoming the most important impediment for commercial ET in this species. Although many factors in the porcine ET-field have been recently evaluated, many others remain to be explored. We investigated here the future reproductive performance of donors and recipients following artificial insemination subsequent to the default surgical embryo recovery approach and to the NsDU-ET procedure, respectively. Although surgical embryo collection did not influence subsequent farrowing rates (90.5\%), litter size decreased severely (8.9 \pm 0.8 piglets) compared to pre-surgery (10.8 \pm 0.3 piglets) and control group (10.7 \pm 0.3 piglets). In contrast, NsDU-ETs did neither affect fertility nor prolificacy of 
recipients in the cycle subsequent to ET, regardless of whether they were pregnant following NsDU-ET or not. These results indicate that while the surgical embryo collection procedure compromises the reproductive future of donor sows, the NsDU-ET approach does not affect the reproductive potential of the recipients after reintroduction to the breeding stock of the farm. Further research is thus needed to improve surgical embryo collection.

\section{Keywords}

Pigs; embryos; non-surgical embryo transfer; surgical embryo collection; future reproductive performance.

\section{Introduction}

Embryo transfer (ET) technology has been a demand of the pig industry for more than 60 years because of its many applications, particularly for the safe exchange of highvalue genetic material. With the recent development of non-surgical deep uterine (NsDU)-ET technology, the commercial use of ET in pigs is now possible. The NsDUET technique is a reliable procedure that yields good fertility levels when coupled to fresh embryos [1-3] or stored embryos, either short-term [4] or long-term [5].

Several factors affecting the success of the NsDU-ET procedure, including superovulation of the donors, synchrony between donors and recipients, use of fresh, short-term or long-term stored embryos, number of embryos transferred per recipient and the recipient's parity, have all been recently assessed [2-6]. However, other factors not directly related to the success of ET have to be quantified to properly estimate the final costs of porcine ET, a most relevant factor for the successful establishment of 
commercial ET programs. An essential component is the impact that embryo collection and/or ET would have on the future reproductive performance of donors and recipients. In pigs, unlike other species, the trans-cervical flushing of the uterus for embryo collection is not effective because of the length and coiled anatomy of the uterine horns [7]. Currently, the collection of embryos from donor sows is only efficient after slaughter or by surgical intervention. Since a common question from genetic companies is the reintroduction of genetically-valuable donors into the breeding stock of the farm after surgical embryo collection, the impact of this procedure on their future reproductive performance must be evaluated.

In contrast, the NsDU-ET procedure involves the insertion of a flexible and thin device into one uterine horn through a conventional insemination catheter previously inserted into the cervical folds as a guide (reviewed in [8]). The NsDU-ET device is able to progress along the uterine lumen, allowing the deposition of the embryos in the middle or anterior quarter of the uterine horn (reviewed in [9]). To the best of our knowledge, there is no information about the effects of NsDU-ET on the reproductive performance of the recipient sows in subsequent cycles, which is also a matter of concern for recipient farms.

The present study aimed to evaluate the effects of surgical embryo collection and NsDU-ET on the subsequent fertility of donors and recipients, respectively, after artificial insemination (AI).

\section{Materials and Methods}

All experimental procedures were performed in accordance with the 2010/63/EU EEC Directive for animal experiments and were reviewed and approved by the Ethical 
Committee for Experimentation with Animals of the University of Murcia, Spain (research code: 638/2012).

\subsection{Animals and farm management}

This work was conducted in field conditions at a commercial farm located in southeastern Spain (Agropor SA, Murcia, Spain). Crossbred sows (Landrace $\times$ LargeWhite) from the same genetic line (1-5 parity), with a lactation period of 21-24 d, were randomly selected at weaning and used as donors or recipients. The females were allocated into individual crates in a mechanically ventilated confinement facility. The semen donors were sexually mature boars (2-3 years of age) of proven fertility, housed in climate-controlled individual pens $\left(20-25^{\circ} \mathrm{C}\right)$ at a commercial breeding boar station for AI-dose semen production in Murcia (Spain). The animals had access to water ad libitum and were fed commercial diets according to their nutritional requirements.

\subsection{Estrus detection in donors and recipients}

Weaning was used to synchronize the estrus between the donors and recipients. Only sows with a weaning-to-estrus interval of 4 to 5 days were selected as donors or recipients. Estrus detection was performed by experienced personnel once a day beginning two days after weaning by allowing snout-to-snout contact of females with a mature boar and by the application of backpressure by experienced personnel. Animals that showed a standing estrous reflex were considered to be in estrus and used in the experiments.

\subsection{Artificial insemination}


The sows were post-cervically inseminated at 0,24 and $36 \mathrm{~h}$ after the onset of estrus. The insemination doses $\left(1.5 \times 10^{9}\right.$ spermatozoa in $\left.45 \mathrm{~mL}\right)$ were prepared from the sperm-rich fractions of the ejaculates, extended in Beltsville thawing solution extender [10] and stored for a maximum of $72 \mathrm{~h}$ at $18^{\circ} \mathrm{C}$.

\subsection{Surgical interventions}

Surgeries were performed in a surgical room located on-farm. Donors were subjected to mid-ventral laparotomy on day 6 of the estrous cycle (day 0: onset of estrus). Animals were sedated by the administration of azaperone ( $2 \mathrm{mg} / \mathrm{kg}$ body weight, intramuscular) and narcosis induced using sodium thiopental (7 mg/kg body weight, intravenous) and maintained with 3-5\% isoflurane. After exposure of the genital tract, corpora lutea were counted on the ovaries, and the embryos were collected as follows; a small incision in the uterine wall $30-40 \mathrm{~cm}$ below the uterotubal junction was performed with a blunt Adson forcep, and then, a glass cannula was inserted through the incision. A volume of $30 \mathrm{~mL}$ of protein-free embryo recovery medium, consisting of Tyrode’s lactate (TL)HEPES-polyvinyl alcohol (PVA) (TL-PVA; [11]) with small modifications [4], was introduced into the uterine horn from the tip of the uterine horn using a $60 \mathrm{~mL}$ syringe connected to a blunt needle. The flushing medium was forced through the glass cannula into a $50 \mathrm{~mL}$ sterile tube by manual massage of the uterus. The incision in the uterus was closed with continuous $2-0$ polyglactin 910 sutures. Then, the uterine horns were placed back inside the abdominal cavity, and the mid-ventral incision was closed with continuous 1 polyglactin 910 sutures in three layers: the peritoneum and linea alba, the adipose layer and the skin. Finally, the incision area was treated with chlorhexidine, and a single intramuscular injection of a long-acting amoxicillin suspension (Clamoxyl $\mathrm{LA}^{\circledR}$; Pfizer, Madrid, Spain) at a dose of $15 \mathrm{mg} / \mathrm{kg}$ was administered. 


\subsection{Non-surgical deep uterine embryo transfer}

All NsDU-ETs were performed in weaned recipients that started estrus 0 or $24 \mathrm{~h}$ after the donors using a previously described method [2-4]. Briefly, six hours prior to ET, each recipient received a single intramuscular injection of a long-acting amoxicillin suspension (Clamoxyl $\mathrm{LA}^{\circledR}$ ) at a dosage of $15 \mathrm{mg} / \mathrm{kg}$ body weight. The recipients were housed in gestation crates in a small room exclusively used for that purpose. The perineal area was thoroughly cleaned with soap and water using a different sponge for each sow. The tail was covered with a latex glove to protect the vulva from possible contamination. The vulva/vestibulum were then washed using sterile gauze soaked with chlorhexidine. Commercial non-surgical ET catheters (Deep Blue ${ }^{\circledR}$ ET catheter, Minitüb, Tiefenbach, Germany) were used. When the catheter was completely inserted into one uterine horn, a one-mL syringe containing 30 embryos in $0.1 \mathrm{~mL}$ of TL-PVA medium was connected to the catheter, and the contents introduced into the catheter. An additional volume of $0.3 \mathrm{~mL}$ of TL-PVA medium was used to force the embryos into the uterus.

\subsection{Experimental design}

The influence of surgical embryo collection and NsDU-ET procedures on the future reproductive potential of donors and recipients, respectively, was established using data registered in an ET program developed recently by the company Agropor SA (Spain) in collaboration with the University of Murcia (Spain). Twenty-five donor sows previously subjected to laparotomy for embryo collection and 41 recipients that were not pregnant following NsDU-ET were artificially inseminated at the subsequent estrus. In addition, a total of 68 recipients that farrowed after NsDU-ET underwent AI in their first estrus post-weaning. 
The control sows within each experimental group [donors $(\mathrm{N}=26)$, non-pregnant recipients $(\mathrm{N}=45)$ and farrowed recipients $(\mathrm{N}=70)$ ], were regular sows inseminated during each experimental period. The number varied due to the variations in the number of experimental sows. Because we tried to use a similar number of experimental and control sows, a determined number of control sows was randomly selected for each experimental group during each experimental period.

From 18 days post-AI, the sows were checked for estrous signs as mentioned above. Pregnancy was diagnosed at days 24-30 post-AI by transcutaneous ultrasonography. All pregnant sows were allowed to carry litters to term, and the farrowing rates, total number of piglets born and number of piglets born alive were recorded. The reproductive data of AIs prior and subsequent to the ET procedure were recorded and statistically compared.

\subsection{Statistical analysis}

The data were analyzed using the IBM SPSS 19 Statistics package (SPSS, Chicago, IL, USA). The percentage data were compared using Fisher's exact test. Continuous variables were evaluated using the Kolmogorov-Smirnov test to assess the assumption of normality, and groups were compared using the unpaired Student's t-test corrected for inequality of variances (Levene's test). When comparison measures were serially made on the same group of animals (i.e., total number of piglets born from previous and subsequent inseminations), a repeated-measures t-test was used. Differences were considered significant when $\mathrm{p}<0.05$. The results are expressed as percentages and means \pm SEM.

\section{Results}


Figure 1 shows the fertility outcomes of the donor sows after AI in their first estrus post-surgery. No differences were found in farrowing rates between previous cycles (93.9\%) and the cycle subsequent to surgical interventions (90.5\%). These results were similar to those recorded for the control sows ( $94.6 \%$ and $91.2 \%$, respectively). In contrast, the total number of piglets born $(8.9 \pm 0.8)$ and the number of piglets born alive (8.6 \pm 0.8$)$ obtained in the farrowing post-surgery were smaller $(\mathrm{P}<0.03)$ than those recorded in the pre-surgery (10.8 \pm 0.3 and $10.5 \pm 0.3$ piglets, respectively) or those of controls (10.7 \pm 0.3 and $10.3 \pm 0.2$ piglets, respectively).

The fertility parameters in the non-pregnant and farrowed recipient groups are shown in Figure 2 and Figure 3, respectively. Within each group, there were no differences in farrowing rates, litter size or total number of piglets born alive between the cycles prior to and subsequent to ET. Additionally, there were also no differences for these variables between the recipient groups and their respective controls. In the non-pregnant recipient group, fertility outcomes were similar between those recipients showing a regular (1824 days) or an irregular (>24 days) return to estrus following ET.

\section{Discussion}

This study is the first to demonstrate that NsDU-ET technology, currently the only available method for nonsurgical transfer of embryos deep into the uterine horn in pigs, does not jeopardize the future reproductive performance of the recipients following conventional AI. On the other hand, our results show that the surgical procedure for embryo collection compromised litter size outcomes (but not farrowing rates) when donors were subsequently bred via AI. The total number of piglets born and number of piglets born alive decreased dramatically (2 piglets per litter) compared with those of the cycles prior to the surgeries and those of the control group. The relevance of these 
findings becomes of utmost significance considering all experiments were conducted under commercial conditions of swine production.

Very little information is, to the best of our knowledge, available regarding how surgical embryo collection impacts the subsequent fertility of donors following conventional AI in pigs. A single study on this topic by James et al. [12] was found, where the authors concluded that multiple surgical embryo recoveries did not seriously affect successive egg collections or the natural farrowing of the donors. However, it must be considered that in that study, the number of donors allowed to carry litters to term was very low and lack control groups, making it difficult to compare its data with our present results. On the other hand, findings in small ruminants make clear that surgical embryo collection negatively affects future fertility [13-14]. Moreover, repeated surgical recovery reduced ovulation and fertilization rates as well as the number of embryos recovered in sheep and goats [15-17]. These findings indicate that the procedure for embryo collection contains elements that diminish ovulation, fertilization rates or numbers of embryos pursuing full development. Surgery implies trauma, of various intensities, to the tip of the uterus (perforation of the wall) as well as manipulation for ovarian/salpingeal/uterine retrieval and exposure, which may cause bleeding and eventual adhesions post-surgery [12]. In small ruminants, post-operative adhesions have been associated with a reduced capture of oocytes, decreased ovulation rate and lower embryo recovery [15-17], all of which compromise future reproductive outcome for the donor. These findings, if extrapolated to pigs, could explain the lack of effect on farrowing rates and the reduced litter size obtained in our study for donor sows following surgical embryo collection. However, extrapolations to pig are difficult to reconcile, considering the anatomical differences between species, particularly considering the anatomy and physiology of the ovarian bursa, e.g. the structure which 
plays a major role in the capture of the newly ovulated oocytes and the facilitation of their inclusion into the infundibulum by the fimbriae.

On the other hand, the recipients represent a substantial cost of an ET program because they are selected from the breeding stock of the recipient farms and must be healthy and in good physical condition with an optimal reproductive history. For these reasons, it is also important to determine their reproductive future after ET so that they can be reintroduced into the breeding stock of the farm. The NsDU-ET catheter can be inserted deep into the uterus in both gilts and sows [1]. In the present study, we only used weaned sows (parity 1-5) as recipients. Several reasons justify this decision. Firstly, the percentages of overall catheter insertions and correct insertions are higher, there is less difficulty in catheter insertion, and the behavior during catheter insertions is better in sows than in gilts $[1,18]$. Secondly, the use of recipient sows was preferred because weaning is a physiological, regular and outstanding process that facilitates estrus synchronization in swine [19]. Thirdly, the parity of the recipient does not affect the success of NsDU-ET [6] and finally, the use of recipient sows with a wide parity range facilitates the scheduling of ET in the recipient farms due to the higher availability onfarm.

One concern with the NsDU-ET procedure is related to the potential damage of the cervix and the uterus mucosa by the NsDU catheter during its advancement along the lumen of the cervical canal and the uterine horn, which could affect not only the recipient's fertility after ET but also its subsequent reproductive performance. Several previous studies indicated that the insertion of the NsDU-ET catheter is non-traumatic (no bleeding during or after insertions), painless and well-tolerated by the recipients, even when the difficulty in inserting the catheter is increased (reviewed in $[8,9,20]$. These data, together with the excellent fertility results following NsDU-ET [2-6], 
suggest that the insertion of the catheter does not cause significant damage to the cervix and/or uterine horn. The results of the present study parallel earlier studies and reinforce the suggestion that NsDU-ET is a non-invasive and welfare-preserving technology. Here, we demonstrated that the fertility and prolificacy of recipients were similar in the cycle subsequent to ET compared to those in the cycles prior to ET, regardless of the pregnancy status after ET. The fertility parameters of the recipients in the cycle subsequent to ET were also similar to those achieved in the control sows. Moreover, when the recipients did not become pregnant following ET, the return-to-estrus interval (regular or irregular) had no influence on their reproductive performance in the subsequent cycle. We speculated that the returns to estrus were due to a failure of the ET procedure or to a loss of all embryos during the peri-implantation period rather than to presumed damage by the ET catheter to the genital tract. Supportive for this speculation is that a return interval to estrus post AI (18-24 days inter estrus interval) results from failure in ovulation or fertilization while a longer return interval ( $>24$ days inter-estrus interval) obeys to embryonic death during implantation [21].

\subsection{Conclusions}

In conclusion, we found that although surgical embryo collection did not interfere with the future farrowing rate of a donor, it negatively affected litter size. We also demonstrated that the recipients can be reintroduced, following a NsDU-ET cycle, into the breeding stock of the farm without losing their reproductive potential. This finding indicates that the potential injury following insertion of the NsDU-ET catheter is nonexistent-to-minimal and that NsDU-ET is a safe reproductive technology that can allow the widespread use of ET in the pig industry. 


\section{Acknowledgments:}

We thank the staff of the Agropor S.A. (Murcia, Spain) piggery for their excellent management of animals. This work was supported by Centro para el Desarrollo Tecnológico e Industrial (CDTI/Agropor SA; IDI-20140140), Madrid, Spain; MINECO-FEDER (AGL2012-38621 and AGL201569735-R), Madrid, Spain; and the Fundación Séneca (19892/GERM/15), Murcia, Spain. We thank MINECO for its grantbased support to CA Martinez and A Nohalez (BES-2013-064087 and BES-2013064069, respectively).

Conflicts of interest

None of the authors have any conflicts of interest to declare.

Author contributions

All authors were involved in all phases of the research and writing of the paper.

\section{References}

[1] Martinez EA, Caamaño JN, Gil MA, Rieke A, Mccauley TC, Cantley TC, et al. Successful nonsurgical deep uterine embryo transfer in pigs. Theriogenology 2004;61:137-46.

[2] Angel MA, Gil MA, Cuello C, Sanzhez-Osorio J, Gomis J, Parrilla I,et al. The effects of superovulation of donor sows on ovarian response and embryo development after nonsurgical deep-uterine embryo transfer. Theriogenology 2014;81:832-9.

[3] Angel MA, Gil MA, Cuello C, Sanzhez-Osorio J, Gomis J, Parrilla I, et al. An earlier uterine environment favors the in vivo development of fresh pig morulae 
and blastocysts transferred by a nonsurgical deep-uterine method. J Reprod Dev 2014;60:371-6.

[4] Martinez EA, Angel MA, Cuello C, Sanchez-Osorio J, Gomis J, Parrilla I, et al. Successful non-surgical deep uterine transfer of porcine morulae after 24 hour culture in a chemically defined medium. PLoS One 2014; 9:e104696.

[5] Martinez EA, Martinez CA, Nohalez A, Sanchez-Osorio J, Vazquez JM, Roca J, et al. Nonsurgical deep uterine transfer of vitrified, in vivo-derived, porcine embryos is as effective as the default surgical approach. Sci Rep 2015;5:10587.

[6] Martinez EA, Nohalez A, Martinez CA, Parrilla I, Vila J, Colina I, et al. The Recipients’ parity does not influence their reproductive performance following non-surgical deep uterine porcine embryo transfer. Reprod Dom Anim 2016;51:123-9.

[7] Martinez EA, Vazquez JM, Roca J, Lucas X, Gil MA, Vazquez JL. Deep intrauterine insemination and embryo transfer in pigs. In: Geisert RD, Niemann H, Doberska C, editors. Control of Pig Reproduction VI. Society of Reproduction and Fertility, Cambridge, UK; 2001. p. 301-11 (Soc. Reprod. Fert. 58:301-311).

[8] Martinez EA, Cuello C, Parrilla I, Rodriguez-Martinez H, Roca J, Vazquez JL, et al. Design, development, and application of a non-surgical deep uterine embryo transfer technique in pigs. Anim Front 2013;3:40-7.

[9] Martinez EA, Gil MA, Cuello C, Sanchez-Osorio J, Gomis J, Parrilla I, et al. Current progress in non-surgical embryo transfer with fresh and vitrified/warmed pig embryos. In: Rodriguez-Martinez H, Soede NM, Flowers WN, editors. Control of Pig Reproduction IX. Leicestershire, UK: Context Products Ltd; 2013. p. 101-12. 
[10] Pursel VG, Johnson LA. Freezing of boar spermatozoa: fertilizing capacity with concentrated semen and a new thawing procedure. J Anim Sci 1975;40:99-102.

[11] Funahashi H, Ekwall H, Rodriguez-Martinez H. Zona reaction in porcine oocytes fertilized in vivo and in vitro as seen with scanning electron microscopy. Biol Reprod 2000;63:1437-42.

[12] James JE, Reeser PD. Embryo recovery in swine. Theriogenology 1979;11:47-50.

[13] Amoah EA, Gelaye S. Biotechnological advances in goat reproduction. J Anim Sci 1997;75:578-85.

[14] Thibier, M., Guerin, B. Embryo transfer in small ruminants: the method of choice for health control in germplasm exchanges. Livestock Prod Sci 2000;62:253-70.

[15] Al-Kamali AA, Boland MP, Crosby TF, Gordon I. Reduced superovulatory response in the ewe following repeated gonadotrophin treatment. Vet Rec 1985; 116:180-1.

[16] Torres S, Sevellec C. Repeated superovulation and surgical recovery of embryos in the ewe. Reprod Nutr Dev 1987;27:859-63.

[17] Cognie Y. State of art in sheep-goat embryo transfer. Theriogenology 1999;51: 105-16.

[18] Cuello C, Berthelot F, Martinat-Botté F, Venturi E, Guillouet P, Vázquez JM, et al. Piglets born after non-surgical deep intrauterine transfer of vitrified blastocysts in gilts. Anim Reprod Sci 2005;85:275-86.

[19] Britt JH, Armstrong JD, Cox NM, Esbenshade KL. Control of follicular development during and after lactation in sows. J Reprod Fert suppl 1985;33: 37 54.

[20] Martinez EA, Cuello C, Parrilla I, Martinez CA, Nohalez A, Vazquez JL et al. Recent advances toward the practical application of embryo transfer in pigs. 
Theriogenology 2016;85:152-61.

[21] Tummaruk P, Lundeheim N, Einarsson S, Dalin A-M. Repeat breeding and subsequent reproductive performance in Swedish Landrace and Swedish Yorkshire sows. Anim Reprod Sci 2001;67:267-80.

\section{Figure captions}

Fig. 1. Farrowing rates and prolificacy of donor sows $(\mathrm{N}=25)$ in the cycles prior to surgical embryo collection and in the subsequent cycle post-surgery. Sows inseminated in the same periods as donors were used as controls $(\mathrm{N}=26)$. Different letters within each period (previous and subsequent cycles) denote differences $(\mathrm{P}<0.02)$ among donors and controls. Asterisks indicate differences $(\mathrm{P}<0.03)$ between previous and subsequent cycles within each group of sows.

Fig. 2. Farrowing rates and prolificacy of non-pregnant recipient sows $(N=41)$ in the cycles prior to non-surgical embryo transfers and in the subsequent cycle to embryo transfer. Sows inseminated in the same periods as recipients were used as controls $(\mathrm{N}=45)$.

Fig. 3. Farrowing rates and prolificacy of farrowed recipient sows $(\mathrm{N}=68)$ in the cycles prior to non-surgical embryo transfers and in the subsequent cycle post-weaning. Sows inseminated in the same periods as recipients were used as controls $(\mathrm{N}=70)$. 